\title{
Síndrome de DRESS desencadeada por carbamazepina: relato de caso
}

Gabriele Denti De Geroni ${ }^{1}$; Fernanda Scortegagna Annes ${ }^{2}$, Karen Regina Rosso Schons ${ }^{3}$

\section{Resumo}

INTRODUÇÃO: A Síndrome de DRESS (Drug Reaction with Eosinophilia and Systemic Symptom) é uma reação severa induzida por drogas, sendo desenvolvida a partir de 3 semanas após o início da terapia farmacológica. Um total de 44 drogas foram descritas como tendo associação com DRESS, sendo as mais frequentemente associadas carbamazepina, fenobarbital, fenitoína, alopurinol, sulfassalazina e nevirapina. $\mathrm{O}$ diagnóstico é feito através dos seguintes critérios: erupção cutânea relacionada à droga, anormalidades hematológicas (eosinofilia/linfocitose atípica), envolvimento sistêmico (adenopatia $\geq 2 \mathrm{~cm}$ de diâmetro, hepatite, nefrite, pneumonite intersticial ou cardite) e febre. $\mathrm{O}$ reconhecimento precoce da síndrome é fundamental, tendo em vista sua mortalidade de 10-20\%. OBJETIVOS: Relatar um caso de Síndrome de DRESS induzida por carbamazepina no HCPF. MÉTODO: Relato de caso. Homem de 19 anos, branco, procedente de Passo Fundo, referindo febre há 15 dias. Inicialmente recebera tratamento empírico com Tamiflu por 7 dias,

\footnotetext{
1 Médica residente em clínica médica no Hospital da Cidade de Passo Fundo. gabidegeroni@gmail.com

2 Médica residente em clínica médica no Hospital da Cidade de Passo Fundo. ferscort@gmail.com

3 Médica dermatologista, mestre em ciências da saúde, professora da faculdade de medicina da Universidade de Passo Fundo. schons.dermatologia@gmail.com
} 
com remissão dos sintomas. Após, reiniciou quadro de febre e iniciou com rash cutâneo, linfonodomegalia cervical, artralgia, mialgia, edema difuso e cefaleia. Previamente epiléptico, utilizava Depakene e Carbamazepina. Exames laboratoriais apresentavam eosinofilia e colestase, com sorologias e demais exames gerais normais. Tomografias evidenciaram proeminência numérica de linfonodos em cadeias cervical posterior e axilar, hepatoesplenomegalia e derrame pleural bilateral de pequenas proporções. Ressonância de crânio e punção lombar inalteradas. Biópsia de lesão cutânea evidenciou dermatite espongiótica de interface com padrão inflamatório perivascular superficial, com presença de componente eosinofílico, entretanto, foi a presença de quatro dos quatro critérios clínicos e laboratoriais da síndrome DRESS que confirmaram o diagnóstico da doença. Introdução do tratamento padrão com corticoterapia (prednisona na dose de $1 \mathrm{mg} / \mathrm{kg} / \mathrm{dia}$ ) e troca da droga suspeita (carbamazepina por topiramato) resultaram em melhora progressiva do quadro com posterior alta hospitalar para acompanhamento ambulatorial. CONCLUSÃO: Devido à raridade dessa reação e à importância do diagnóstico rápido, é necessário o reconhecimento precoce da síndrome e a cessação da droga causal. Corticóide oral é mantido por pelo menos 6-8 semanas para evitar recidiva. Se os sintomas persistirem, apesar da terapia, pode-se considerar o uso de imunoglobulina EV, rituximab e valganciclovir.

Palavras-chave: DRESS; eosinofilia; carbamazepina 


\section{Referências}

CACOUB, P. The DRESS Syndrome: A Literature Review. The American Journal of Medicine, 588-597, Jul 2011

LOBO, Ines et al. ERUPÇÃO A FÁRMACO COM EOSINOFILIA E SINTOMAS SISTÉMICOS (Síndrome DRESS). Acta Med Port 2008; 21: 367-372

Lodi, C.M.; Bevilaqua, M.; Boreli, N.S.; Brotas, A.; Osório, D.A.; Sindrome De DRESS Desencadeado Por Carbamazepina: Relato De Caso E Revisão De Literatura. In: II Congresso Brasileiro de Medicina Hospitalar - II CBMH [= Blucher Medical Proceedings, vol.1, num.5] São Paulo: Editora Blucher, 2014. p.16'

SURAN, L.F. Drug-reaction eosinophilia and systemic symptoms and drug-induced hypersensitivity syndrome. Australasian Journal of Dermatology, Australia, 15-23, 30 Abril 2013 